\title{
Microlitiasis testicular como factor de riesgo de Cáncer testicular - Estudio de casos y controles
}

\section{Testicular Microlithiasis: A Risk Factor for Testicular Cancer - A Case Control Study}

\author{
Hugo López-Ramos ${ }^{1}$ Mauricio Medina-Rico ${ }^{2}$ Sofía Manjarrés ${ }^{3}$ Maria Camila Usubillaga ${ }^{4}$ \\ Daniela Sierra ${ }^{5}$
}

${ }^{1}$ Departamento de Urología. Hospital Universitario San Ignacio. Facultad de Medicina, Pontificia Universidad Javeriana, Bogotá, Colombia

${ }^{2}$ Departamento de Epidemiología Clínica y Bioestadística. Hospital Universitario San Ignacio. Facultad de Medicina, Pontificia Universidad Javeriana, Bogotá, Colombia

${ }^{3}$ Hospital Universitario San Ignacio. Facultad de Medicina, Pontificia Universidad Javeriana, Bogotá, Colombia

${ }^{4}$ Hospital Universitario San Ignacio. Facultad de Medicina, Pontificia Universidad Javeriana, Bogotá, Colombia

${ }^{5}$ Hospital Universitario San Ignacio. Facultad de Medicina, Pontificia Universidad Javeriana, Bogotá, Colombia

\begin{abstract}
Address for correspondence Mauricio Medina-Rico, Departamento de Epidemiología Clínica y Bioestadística, Pontificia Universidad Javeriana, Cr. 7ª. \# 40-62 piso $2^{\circ}$ Hospital Universitario de San Ignacio, Bogotá, Colombia (e-mail: medina.mauricio@javeriana.edu.co).
\end{abstract}

Urol Colomb 2020;29:39-42.

\section{Resumen}

\section{Palabras clave}

- cáncer testicular

- neoplasias testiculares

- enfermedades testiculares

- microlitiasis testicular

- odds ratio

- factor de riesgo
Introducción Con una prevalencia entre el 1,5\% y el 5,6\%, la microlitiasis testicular es un hallazgo incidental. Aunque se ha propuesto una posible asociación entre la microlitiasis testicular y el cáncer testicular dicha relación no ha sido comprobada.

Objetivos Establecer la relación entre microlitiasis testicular y cáncer testicular.

Métodos Se realizó un estudio de casos y controles con la base de datos de un hospital de la ciudad de Bogotá de pacientes atendidos entre 2007 y 2017. Tanto casos como controles debían tener entre 5 y 35 años de edad, sin otros antecedentes que aumentaran el riesgo de cáncer testicular (criptorquidia, antecedente de cáncer testicular contralateral). Los casos debían tener reporte ecográfico y patológico del cáncer testicular así como una historia clínica completa de valoración por Urología. Los controles fueron pacientes que consultaron por otros cuadros de molestia testicular a quienes se les descartó ecográficamente la presencia de cáncer.

Resultados Se identificaron 24 casos y 96 controles. Al momento de calcular los riesgos relativos indirectos, se obtuvieron un OR crudo de $7,86(95 \% \mathrm{Cl} 2,3-26,86)$ y un OR ajustado por tabaquismo de 10,66 (95\% IC 3,29 - 34,55). Al ajustar por edad, historia de cáncer familiar y antecedente de varicocele, no se encontraron diferencias.

Conclusión Existe una aparente asociación entre microlitiasis testicular y cáncer testicular. A pesar de eso, estudios con tamaños de muestra más grande serían beneficiosos. Así mismo, al no poder determinar la existencia de una asociación received

December 18, 2018

accepted

March 19, 2019
DOI https://doi.org/

10.1055/s-0039-1688464.

ISSN 0120-789X.

e ISSN 2027-0119.
Copyright ( 2020 , Sociedad Colombiana License terms de Urología. Publicado por Thieme Revinter Publicações Ltda., Rio de Janeiro, Brazil. Todos los derechos reservados.

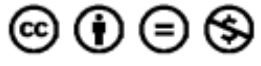




\begin{abstract}
Keywords

- testicular cancer

- testicular neoplasm

- testiculares diseases

- testicular microlithiasis

- odds ratio

- risk factor

Introduction Testicular microliathiasis is an incidental finding with a prevalence that varies between $1,5 \%$ and $5,6 \%$. Although a possible association between testicular microlithiasis and testicular cancer has been proposed, this relationship has not been proven.

Objective To establish the association between testicular microlithiasis and testis cancer.

Methods A case-control study was conducted with the database of a hospital in the city of Bogotà, Colombia, of patients treated between 2007 and 2017. Both cases and controls should be between 5 and 35 years of age, with no other history that would increase the risk of testicular cancer (cryptorchidism, antecedent of contralateral testicular cancer). The cases had to have an ecographic and pathological report of testicular cancer as well as a complete clinical history of assessment by Urology. Likewise, the control group were patients who attended during the same period, with other testicular problems, such as: hydrocele, varicocele or inguinal hernia and with ultrasound findings that ruled out testicular cancer.

Results We identified 24 cases and 96 controls. We found a crude OR of $7.86(95 \% \mathrm{Cl}$ 2.3-26.86) and an OR adjusted for smoking of 10.66 (95\% Cl 3.29-34, 55). When adjusting for age, family cancer history and varicocele history, no differences were found.

Conclusion There is an apparent association between testicular microlithiasis and testicular cancer. Despite this, studies with larger sample sizes would be beneficial. Likewise, since it cannot determine the existence of a temporary association that allows to speak of causality between the two conditions, these results must be taken with caution.
\end{abstract}

temporal que permita hablar de causalidad entre las dos condiciones, esos resultados deben tomarse con cautela.

\section{Introducción}

El cáncer testicular es un cáncer poco frecuente entre las patologías tumorales con una incidencia que varía entre países llegando a ser del 2,6/100.000 personas al año en Sur América y de 7,8/100.000 personas al año en Europa. ${ }^{1}$ Si bien la confirmación diagnóstica se realiza por el estudio patológico de la pieza quirúrgica, la ecografía es considerada la prueba de elección para identificar tumores testiculares. ${ }^{2,3} \mathrm{Al}$ igual que su incidencia, la mortalidad del cáncer testicular es baja, con tasas de mortalidad de hasta 0,3/100.000 habitantes. ${ }^{1}$ El cáncer testicular es un tumor con buen pronóstico, principalmente por la posibilidad de un tratamiento oportuno cuando llega a realizarse una detección temprana. ${ }^{4} \mathrm{~A}$ pesar de eso, en países como Colombia, se suele retrasar el diagnóstico alrededor de 5 meses por diferentes elementos como lo son las barreras culturales y sociales que disminuyen la consulta de los hombres a los servicios médicos. ${ }^{5}$

Por su parte, la microlitiasis testicular es una condición asintomática usualmente diagnosticada como un hallazgo incidental por medio de ultrasonografía (US) testicular y su etiología aún no está clara. ${ }^{6}$ A nivel imagenológico, la microlitiasis testicular se describe como múltiples focos de mayor ecogenicidad dentro del parénquima testicular de 1$3 \mathrm{~mm}$ de tamaño. ${ }^{7}$ Aunque el reporte de microlitiasis testicular ha aumentado con los años, secundario a los avances tecnológicos mediante las ecografías de alta resolución que han permitido una visión más precisa del parénquima testicular, la incidencia no es clara. ${ }^{8}$ Sin embargo, algunos estudios han estimado una prevalencia que varía entre el $1,5 \%$ y el 5,6\% en adultos jóvenes sanos. ${ }^{9-12}$

Aunque se han sugerido clasificaciones de la microlitiasis testicular según hallazgos en las US como la de Sanli o et al (2008), eso no han demostrado que estén relacionadas con el desarrollo del cáncer testicular. ${ }^{13}$ Esa cuestión concuerda con algunos estudios que no han encontrado relación entre la microlitiasis testicular y el cáncer testicular. ${ }^{14}$ No obstante, otros autores han sugerido una posible asociación entre ambas condiciones ante una mayor frecuencia de microlitiasis en pacientes con cáncer testicular. ${ }^{15}$

Ante las diferentes posiciones, se hace necesario estudiar más la posible asociación enre microlitiasis testicular y el cáncer testicular, así como poder identificar otros elementos que pueden ayudar a alertar el posible desarrollo de cáncer testicular agilizando así el diagnóstico temprano para que se conserven los buenos pronósticos mencionados anteriormente. El propósito de este estudio es determinar si la microlitiasis testicular se puede considerar un factor de riesgo para el desarrollo de cáncer testicular, y por tanto, generar algunas recomendaciones para el seguimiento de los pacientes a 
quienes se les reporte microlitiasis testicular y mejorarles el pronóstico en caso de un posible desarrollo de la enfermedad.

\section{Métodos}

Se realizó un estudio de casos y controles con la base de datos de un hospital de cuarto nivel de Bogotá, Colombia tomando pacientes que consultaron al hospital entre 2012 y 2017 al servicio de urgencias o urología. Para la elección de los casos, se tuvieron en cuenta pacientes con diagnóstico de cáncer testicular identificado mediante ecografía testicular, y con reporte patológico posterior a la orquiectomía que incluyera cualquier variante histológica del cáncer testicular. Los controles fueron pacientes que consultaron hidrocele, varicocele, epididimitis, sospecha de cáncer testicular, y en general por cualquier cuadro de molestia testicular a quienes se les realizó ecografía testicular intrainstitucional y cuyo reporte rechazara la existencia de posibles lesiones tumorales. Tanto en casos como en controles se buscó en la historia clínica la presencia microlitiasis en el reporte de la ecografía testicular y se identificaron otros posibles factores de riesgo como tiempo entre en diagnóstico de la microlitiasis y el desarrollo de cáncer testicular, tipo histológico, edad al momento del diagnóstico, y antecedente de tabaquismo.

Los sujetos debían ser hombres entre 5 y 35 años, con historia clínica por urología con antecedentes completos. Se excluyeron aquellos pacientes con factores que podían aumentar el desarrollo de cáncer testicular o podían generar lesiones del parénquima tales como antecedente de criptorquidea, trauma o torsión testicular y antecedente de cáncer testicular contralateral. Se calculó un tamaño de muestra de 24 casos y 96 controles acorde a un estimado de microlitiasis testicular del $5 \%$ de la población general y un aproximado del $30 \%$ de pacientes con cáncer testicular para realizar un análisis 1 a 4 . Finalmente, el análisis se realizó mediante el programa Stata 12 con el cual se calculó el odds ratio (OR) crudo, así como el OR ajustado mediante regresión logística y estratificación de las posibles variables de confusión.

\section{Resultados}

Un total de 120 sujetos fueron incluidos en el estudio, 24 casos con cáncer testicular y 96 controles. La edad media de los casos fue de 23,92 años y de los controles 23,41 años. De los casos los tipos de cáncer más frecuente fueron los de composición mixta (carcinoma embrionario más teratoma), seguido del seminoma puro y los carcinomas embrionarios. Respecto de la microlitiasis testicular, ella pudo observarse en el $41,7 \%$ de los casos y en el 8,3\% de los controles. Ninguno de los pacientes (casos o controles), se encontraban en estudio de infertilidad ( $\mathbf{- T a b l a ~} \mathbf{1}$ ).

El riesgo relativo indirecto (OR) crudo o chance de tener cáncer testicular ante el hallazgo microlitiasis fue de 7,86 (IC95\% 2,3-26,86). Al estratificar por edad, antecedente familiar de cáncer o de varicocele, no se encontraron diferencias en los OR y mediante la prueba de MaentelHaneszel se concluyó que no había interacción ni confusión
Tabla 1 Características demográficas de los sujetos

\begin{tabular}{|c|c|c|c|c|}
\hline & \multicolumn{2}{|c|}{ Casos } & \multicolumn{2}{|c|}{ Controles } \\
\hline & $n$ & $\%$ & $n$ & $\%$ \\
\hline \multicolumn{5}{|l|}{ Edad } \\
\hline $5-20$ años & 6 & 25 & 29 & 30,2 \\
\hline 21 - 35 años & 18 & 75 & 67 & 69,8 \\
\hline \multicolumn{5}{|l|}{ Tabaquismo } \\
\hline Sí & 8 & 33,3 & 12 & 12,5 \\
\hline No & 16 & 66,7 & 84 & 87,5 \\
\hline \multicolumn{5}{|c|}{ Historia Familiar de cáncer } \\
\hline Sí & 6 & 25 & 4 & 4,2 \\
\hline No & 18 & 75 & 92 & 95,8 \\
\hline \multicolumn{5}{|l|}{ Varicocele } \\
\hline Sí & 4 & 16,7 & 18 & 18,8 \\
\hline No & 20 & 83,3 & 78 & 81,3 \\
\hline \multicolumn{5}{|l|}{ Microlitiasis testicular } \\
\hline Sí & 10 & 41,7 & 8 & 8,3 \\
\hline No & 14 & 58,3 & 88 & 91,7 \\
\hline \multicolumn{5}{|l|}{ Histología } \\
\hline Carcinoma embrionario & 6 & 25 & - & - \\
\hline Seminoma & 6 & 25 & - & - \\
\hline Teratoma & 3 & 12,5 & - & - \\
\hline Mixto & 9 & 37,5 & - & \\
\hline
\end{tabular}

Tabla 2 Cálculo de los riesgos indirectos (OR) por las variables evaluadas

\begin{tabular}{|l|l|l|}
\hline & OR & IC 95\% \\
\hline Crudo & 7,86 & $2,29-26,86$ \\
\hline Ajustado a tabaquismo & 10,66 & $3,29-34,55$ \\
\hline Ajustado por edad & 7,87 & $2,64-23,41$ \\
\hline Ajustado por cáncer familiar & 7,66 & $2,45-23,96$ \\
\hline Ajustado por varicocele & 8,01 & $2,6-24,6$ \\
\hline
\end{tabular}

por esas variables. De manera diferente, el tabaquismo evidenció un OR de 10,66 (IC95\% 3,29-34,55) (-Tabla 2).

\section{Discusión}

Nuestro estudio evidencia un OR elevado de presentar cáncer testicular ante la presencia de microlitiasis testicular, resultados similares a otros estudios similares con poblaciones incluso más grandes que la nuestra. ${ }^{16}$ Así mismo, se encontraron dos metaanálisis las cuales apoyan la teoría de una posible asociación entre la presentación de microlitiasis testicular y cáncer testicular. $^{15,17}$

Cuando se consideraron las otras variables del estudio, se observó un aumento del riesgo relativo indirecto cuando se ajustó por tabaquismo. Dicho hallazgo se asemeja al de los resultados de estudios como el de Srivastava A et al (2004), que 
han identificado al cigarrillo como un factor de riesgo para el desarrollo de cáncer testicular. ${ }^{18}$ Por otro lado, aunque nuestro estudio no identificó que la historia de cáncer familiar genere un mayor riesgo en el desarrollo de cáncer testicular, algunos estudios como los de Kratz CP et al (2011) o Landero-Huerta Da et al (2017), han descrito la existencia de algunos genes asociados al desarrollo de cánceres testiculares (principalmente para tumores de células germinales), ${ }^{19,20}$ así mismo, algunos como Zhan L et al (2018) han sugerido una asociación entre historia de cáncer familiar con tumores testicular no seminomas. ${ }^{21}$

Finalmente, al ajustar por edad no se generó ninguna diferencia en el riesgo de desarrollo de cáncer testicular por la microlitiasis. Por otro lado, al ajustar por varicocele si bien él generó un ligero aumento en el riesgo respecto el OR crudo, dicha diferencia no pareció ser significativa.

Nuestros resultados deben ser tomados con cautela dado que el reporte de microlitiasis se hace en paralelo con el reporte de cáncer testicular, por lo cual a nivel de causalidad no podemos estar seguros de la asociación temporal entre esas dos condiciones. Con eso en mente, algunos estudios de corte prospectivo que han evaluado la microlitiasis testicular no han logrado evidenciar una asociación definida de presentar cáncer testicular y que por ende, esa población no debería ser seguida a lo largo del tiempo por lo menos a nivel de imágenes radiológicas. 22,23 A pesar de eso, otros autores han propuesto que esa población debería ser seguida al menos mediante el autoexamen testicular y para ello se les debería realizar un proceso de educación durante la consulta siendo eso aún más importante en caso de presentar otros posibles factores de riesgo como lo sería el tabaquismo. ${ }^{24-26}$

\section{Conclusiones}

Los pacientes con microlitiasis testicular, podrían llegar a tener un mayor riesgo de desarrollar cáncer testicular, no obstante, la asociación entre las dos condiciones no es concluyente, por lo cual se debe ser cauteloso al momento de interpretar esos resultados para no alarmar a los pacientes que pueden llegar a ser diagnosticados con microlitiasis testicular. Sin embargo, vale la pena educar a esa población en términos del autoexamen para ser realizado al menos una vez al año siendo eso más importante en caso de presentar otros factores de riesgo.

\section{Conflicto de Intereses}

Los autores declaran no tener ningún conflicto de intereses.

\section{Referencias}

1 Medina-Rico M, López-Ramos H. Testicular cancer epidemiology in developing countries. Review of the literature. Arch Esp Urol 2017;70(05):513-523

2 Baird DC, Meyers GJ, Hu JS. Testicular Cancer: Diagnosis and Treatment. Am Fam Physician 2018;97(04):261-268

3 Stevenson SM, Lowrance WT. Epidemiology and Diagnosis of Testis Cancer. Urol Clin North Am 2015;42(03):269-275

4 Albers P, Albrecht W, Algaba F, et al; European Association of Urology. EAU guidelines on testicular cancer: 2011 update. Eur Urol 2011;60(02):304-319
5 Correa Ochoa JJ, Velásquez Ossa D, Lopera Toro AR, Martínez González CH, Yepes Pérez A. Guía colombiana de cáncer de testículo. Urol Colomb. 2016;25(03):274-285

6 Shanmugasundaram R, Singh JC, Kekre NS. Testicular microlithiasis: Is there an agreed protocol? Indian J Urol 2007; 23(03):234-239

7 Meissner A, Mamoulakis C, de la Rosette JJ, Pes MPL. Clinical update on testicular microlithiasis. Curr Opin Urol 2009;19(06):615-618

8 Tan M-H, Eng C. Testicular microlithiasis: recent advances in understanding and management. Nat Rev Urol 2011;8(03): 153-163

9 van Casteren NJ, Looijenga LHJ, Dohle GR. Testicular microlithiasis and carcinoma in situ overview and proposed clinical guideline. Int J Androl 2009;32(04):279-287

10 Serter S, Gümüş B, Unlü $M$, et al. Prevalence of testicular microlithiasis in an asymptomatic population. Scand J Urol Nephrol 2006;40(03):212-214

11 Goede J, Hack WWM, van der Voort-Doedens LM, Sijstermans K, Pierik FH. Prevalence of testicular microlithiasis in asymptomatic males 0 to 19 years old. J Urol 2009;182(04):1516-1520

12 Derogee M, Bevers RF, Prins HJ, Jonges TG, Elbers FH, Boon TA. Testicular microlithiasis, a premalignant condition: prevalence, histopathologic findings, and relation to testicular tumor. Urology 2001;57(06):1133-1137

13 Sanli O, Kadioglu A, Atar M, Acar O, Nane I, Kadioglu A. Grading of Classical Testicular Microlithiasis Has No Effect on the Prevalence of Associated Testicular Tumors. 2008;80(03):310-316

14 Pedersen MR, Rafaelsen SR, Møller H, Vedsted P, Osther PJ. Testicular microlithiasis and testicular cancer: review of the literature. Int Urol Nephrol 2016;48(07):1079-1086

15 Wang T, Liu L, Luo J, Liu T, Wei A. A Meta-Analysis of the Relationship between Testicular Microlithiasis and Incidence of Testicular Cancer. Urol J 2015;12(02):2057-2064

16 Trout AT, Chow J, McNamara ER, et al. Association between Testicular Microlithiasis and Testicular Neoplasia: Large Multicenter Study in a Pediatric Population. Radiology 2017; 285(02):576-583

17 Suominen JS, Jawaid WB, Losty PD. Testicular microlithiasis and associated testicular malignancies in childhood: a systematic review. Pediatr Blood Cancer 2015;62(03):385-388

18 Srivastava A, Kreiger N. Cigarette smoking and testicular cancer. Cancer Epidemiol Biomarkers Prev 2004;13(01):49-54

19 Kratz CP, Han SS, Rosenberg PS, et al. Variants in or near KITLG, BAK1, DMRT1, and TERT-CLPTM1L predispose to familial testicular germ cell tumour. J Med Genet 2011;48(07):473-476

20 Landero-Huerta DA, Vigueras-Villasenor RM, Yokoyama-Rebollar E, et al. Epigenetic and risk factors of testicular germ cell tumors: a brief review. Front Biosci 2017;22:1073-1098

21 Zhang L, Yu H, Hemminki O, Försti A, Sundquist K, Hemminki K. Familial Associations in Testicular Cancer with Other Cancers. Sci Rep 2018;8(01):10880

22 Patel KV, Navaratne S, Bartlett E, et al. Testicular Microlithiasis: Is Sonographic Surveillance Necessary? Single Centre 14 Year Experience in 442 Patients with Testicular Microlithiasis. Ultraschall Med 2016;37(01):68-73

23 Marte A, Pintozzi L, Cretì G, et al. Long-Term Follow-Up of Testicular Microlithiasis in Children and Adolescents: Multicenter Prospective Cohort Study of the Italian Society of Pediatric Urology. Eur J Pediatr Surg 2017;27(02):155-160

24 Yesil S, Tanyildiz HG, Sahin G. How should we monitor boys with testicular microlithiasis? Pediatr Hematol Oncol 2016;33(03): 171-177

25 Kola B Pediatric Testicular Microlithiasis: To Refer or Not to Refer? Glob Pediatr Heal. 2017;4:2333794 × 17731854 .

26 Price NR, Charlton A, Simango I, Smith GHH. Testicular microlithiasis: the importance of self-examination. J Paediatr Child Health 2014;50(10):E102-E105 